\title{
ON ABSOLUTELY CONVERGENT EXPONENTIAL SUMS $\left({ }^{1}\right)$
}

\author{
BY \\ LEON BROWN, ALLEN SHIELDS AND KARL ZELLER
}

Consider the following problem. Is it possible to represent zero by an absolutely convergent series of exponentials with bounded exponents:

$$
\sum_{1}^{\infty} a_{n} e^{\alpha_{n} z} \equiv 0, \quad 0<\sum\left|a_{n}\right|<\infty, \quad\left|\alpha_{n}\right|<M \quad(n \geqq 1)
$$

where $\left\{a_{n}\right\}$ and $\left\{\alpha_{n}\right\}$ are complex numbers and the $\alpha_{n}$ are all distinct? Equation (1) is equivalent to

$$
\sum_{1}^{\infty} \frac{a_{n}}{z-\alpha_{n}} \equiv 0 \quad(|z|>M), \quad 0<\sum\left|a_{n}\right|<\infty, \quad\left|\alpha_{n}\right|<M,
$$

since (2) is the Borel-Laplace transform of (1) (see $\$ 1$ ).

To our knowledge the solution to this problem was first given in 1921 by $\mathrm{J}$. Wolff [21] who gave an example of such a representation of zero.

In this paper we consider the problem of characterizing those bounded complex sequences $\left\{\alpha_{n}\right\}$ for which coefficients $\left\{a_{n}\right\}$ exist such that (1) holds. In $\S 2$ a number of preliminary results are given. For example, (1) is impossible if the closure of the set $\left\{\alpha_{n}\right\}$ contains no interior and does not separate the plane, or if all the $\alpha_{n}$ lie in a convex domain and at least one, with a nonzero coefficient, lies on the boundary.

In $\S 3$ we obtain a characterization of those $\left\{\alpha_{n}\right\}$ for which (1) is possible, under the assumption that the $\alpha_{n}$ lie in the unit circle and have no interior limit points. The condition is that almost every boundary point $\exp (i \theta)$ be approachable nontangentially (i.e. inside of an angle) by a subsequence of $\left\{\alpha_{n}\right\}$. This is equivalent to

$$
\sup _{n}\left|f\left(\alpha_{n}\right)\right|=\sup _{|z|<1}|f(z)|
$$

for every bounded analytic $f$ in the unit circle.

In $\S 4$ we study the set $E(G)$ of all those entire functions $h(z)$ admitting a representation

$$
h(z)=\sum a_{n} e^{\alpha_{n} z}, \quad \sum\left|a_{n}\right|<\infty, \quad \alpha_{n} \in G,
$$

Presented to the Society, January 20, 1959 and April 17, 1960; received by the editors October 9, 1959.

(1) A portion of this work was done while the second author held a Rackham Foundation summer research grant from the University of Michigan. 
where $G$ is a Jordan domain. With a suitable norm $E(G)$ is a Banach space whose conjugate space is the space of all bounded analytic functions in $G$ with the supremum norm. In case $G$ is the unit circle we obtain a characterization of $E$ as a quotient space of $L_{1}(0,2 \pi)\left(E=L_{1} / H_{1}\right)$. This includes Wolff's result [21] that $E$ contains all entire functions of exponential type less than 1. It also gives a representation of Fourier coefficients as solutions of infinite "Vandermonde" systems of equations.

In $\$ 5$ we give some additional properties of the space $E$.

We wish to thank Professors G. Lorentz, G. Piranian, P. C. Rosenbloom, and J. L. Ullman for their helpful suggestions and encouragement.

1. Background material. We shall need a few standard results on entire functions of exponential type, and on bounded analytic functions in the unit circle. These are stated here, proofs may be found in Boas [3, Chapter 5] and Privaloff $[15$, Chapter 1$]$.

Let $h(z)=\sum a_{n} z^{n} / n$ ! be an entire function and let $M(r)$ denote the maximum of its modulus on a circle of radius $r$. The function $h$ is said to be of exponential type 1 if

$$
M(r)=O\left(e^{(1+\epsilon) r}\right)
$$

for every $\epsilon>0$.

The function $H(z)=\sum a_{n} / z^{n+1}$ is called the Borel transform (or BorelLaplace transform) of $h$. The series for $H$ converges for $|z|>1$, and vanishes at $z=\infty$. One may recover $h$ as an integral

$$
h(z)=\frac{1}{2 \pi i} \int_{|w|-r} e^{z w} H(w) d w
$$

for every $r>1$. The Borel transform establishes a one-to-one correspondence between the class of all entire functions of exponential type 1, and the class of all functions analytic for $|z|>1$ and vanishing at infinity.

Let $H_{\infty}$ denote the class of all bounded analytic functions in the unit circle. It is a Banach space under the supremum norm. Each $f \in H_{\infty}$ has a radial limit

$$
f\left(e^{i t}\right)=\lim _{r \rightarrow 1^{-}} f\left(r e^{i t}\right)
$$

at almost every boundary point $p=\exp (i t)$, and the resulting boundary function is a bounded measurable function whose essential supremum equals $\|f\|$. In addition, whenever the radial limit exists, the limit exists and has the same value inside of any angle with vertex at $p$. The radial limit can be zero only on a set of measure zero, unless $f \equiv 0$.

Let $\left\{\alpha_{n}\right\}$ be a sequence of points inside the unit circle. The necessary and sufficient condition that there exist an $f \in H_{\infty}$ vanishing at the $\alpha_{n}$ but nowhere else inside the unit circle is that $\sum\left(1-\left|\alpha_{n}\right|\right)<\infty$. 
2. Preliminary results. Most of the results of this section apply to a more general representation of zero than is given in (1). Let $\mu$ be any finite, complex-valued Borel measure of compact support $K$ in the plane. We say that $\mu$ represents zero if

$$
\int e^{z w} d \mu(w) \equiv 0
$$

Lemma 1. Equation (1') holds if and only if

$$
\int P(w) d \mu(w)=0
$$

for all polynomials $P$.

Proof. Differentiating $\left(1^{\prime}\right) n$ times and evaluating at $z=0$ we obtain $\int w^{n} d \mu(w)=0$, and (5) follows. Conversely, (5) implies $\left(1^{\prime}\right)$ since $f_{z}(w)=e^{z w}$ may be uniformly approximated by polynomials on the support of $\mu$.

Instead of using the exponential function in this lemma we could have used any entire function $g$ that, together with all its derivatives, is different from zero at the origin. Equation $\left(1^{\prime}\right)$ then becomes: $\int g(z w) d \mu(w) \equiv 0$. This remark applies to several of the following lemmas.

THEOREM 1. If $K$ contains no interior points and does not separate the plane, and if $\left(1^{\prime}\right)$ holds, then $\mu=0$.

Proof. By a theorem of Lavrentieff (see Mergelyan, [13, Chapter 1, §4]) polynomials are uniformly dense in the space $C(K)$ of all continuous, complex-valued functions on $K$. Hence by Lemma $1, \int f d \mu=0$ for all $f \in C(K)$, and so $\mu \equiv 0$.

Let $G$ be a Jordan region (the interior of a simple closed Jordan curve). We shall say that a measure $\mu$ is "in" $G$ if $\mu(E)=0$ for all sets $E$ disjoint from $G$. This is not quite the same as saying that the support of $\mu$ is contained in $G$. (The support of $\mu$ is the smallest closed set $K$ such that $\mu(E)=0$ for all $E$ disjoint from $K$.) Thus in (1) $\mu$ is a sequence of mass points; $\mu$ is in the circle $|z|<M$ but the support of $\mu$ is $\left\{\alpha_{n}\right\}-$ (the closure of the set $\left\{\alpha_{n}\right\}$ ).

Let $H_{\infty}(G)$ denote the set of all bounded analytic functions in $G$. If $G$ is the interior of the unit circle we write simply $H_{\infty}$ as in $\S 1$.

LEMMA 2. If $\mu$ is a measure in the Jordan region $G$ and if ( $\left.1^{\prime}\right)$ holds then

$$
\int f(w) e^{z w} d \mu(w) \equiv 0
$$

for all $f \in H_{\infty}(G)$.

Proof. If $f$ is a polynomial the result follows as in Lemma 1 by differentiating $\left(1^{\prime}\right)$. If $f$ is the uniform limit of polynomials the result follows from a 
passage to the limit. Finally, if $f$ is a bounded pointwise limit of functions for which (6) holds, then by the Lebesgue bounded convergence theorem, (6) will hold for $f$.

Now let $f \in H_{\infty}(G)$ and let $\phi$ be a Riemann mapping function taking the unit circle $(|\zeta|<1)$ onto $G$. Then $g(\zeta)=f(\phi(\zeta)) \in H_{\infty}$. Let $g_{r}(\zeta)$ $=g(r \zeta)(0<r<1)$. Then $g_{r}$ is analytic for $|\zeta|<1 / r$, and in particular $g_{r}$ is continuous for $|\zeta| \leqq 1$. It is known (see Bieberbach [2, p. 33]) that $\phi$ maps $|\zeta| \leqq 1$ homeomorphically onto $G^{-}$, and therefore $f_{r}(z)=g_{r}\left(\phi^{-1}(z)\right)$ is analytic in $G$ and continuous on $G^{-}$. Hence by a theorem of Walsh [19] $f_{r}$ is the uniform limit of polynomials on $G^{-}$. The result now follows since $f$ is the bounded pointwise limit of $f_{r}$ as $r \rightarrow 1$.

Lemma 3. If $\mu$ is a measure in the Jordan region $G$ then $\left(1^{\prime}\right)$ holds if and only if

$$
\int f(w) d \mu(w)=0
$$

for every $f \in H_{\infty}$.

This follows immediately from Lemmas 1 and 2 . We do not know to what extent it holds for more general regions.

CoRollary. If $\mu$ is a measure in the Jordan region $G$ for which $\left(1^{\prime}\right)$ holds and if $\phi$ is any analytic function mapping $G$ into itself, then

$$
\int e^{z \phi(w)} d \mu(w) \equiv 0 \text {. }
$$

Proof. We define a new measure $\sigma$ by: $\sigma(E)=\mu\left(\phi^{-1}(E)\right)$ for all Borel sets $E$. One verifies easily that

$$
\int f(w) d \sigma(w)=\int f(\phi(w)) d \mu(w)
$$

for all continuous $f$. Now let $f \in H_{\infty}(G)$. Then $f(\phi(w)) \in H_{\infty}(G)$ and so the right side of (9) vanishes by Lemma 3 . Thus $\int f d \sigma=0$ for all $f \in H_{\infty}(G)$, and so by the other half of Lemma $3,\left(1^{\prime}\right)$ holds for $\sigma$. But this is precisely equation (8) which was to be proven.

The last two results of this section deal with equation (1) rather than with $\left(1^{\prime}\right)$. The first result enables us to add one more term to a given representation of zero.

Lemma 4. Let (1) hold, except that instead of the condition $\left|\alpha_{n}\right|<M$ we assume that the exponents all lie in a Jordan region $G$ and have no interior limit points. Let $\alpha \in G$. Then coefficients $\left\{b_{n}\right\}$ exist such that

$$
\exp (\alpha z)+\sum b_{n} \exp \left(\alpha_{n} z\right) \equiv 0, \quad \sum\left|b_{n}\right|<\infty .
$$


Proof. We may assume $\alpha \neq \alpha_{n}(n=1,2, \cdots)$ for otherwise (10) is trivial. If we differentiate (1) $k$ times and evaluate at $z=0$ we obtain

$$
\sum_{n=1}^{\infty} a_{n} \alpha_{n}^{k}=0 \quad(k=0,1,2, \cdots) .
$$
by

Let now $b_{n}{ }^{\prime}=a_{n} /\left(\alpha_{n}-\alpha\right), b^{\prime}=-\sum b_{n}{ }^{\prime}$, and define the functions $h(z), \phi(z)$

$$
h(z)=\sum b_{n}^{\prime} \exp \left(\alpha_{n} z\right), \quad \phi(z)=b^{\prime} \exp (\alpha z)+h(z) .
$$

From (11) we see that $\phi$ and all its derivatives vanish at the origin and thus $\phi \equiv 0$. If $b^{\prime} \neq 0$ we have (10) and the proof is complete.

Now suppose $b^{\prime}=0$. From (12) we see that $h \equiv 0$. We repeat the process, letting $b_{n}^{\prime \prime}=b_{n}^{\prime} /\left(\alpha_{n}-\alpha\right)$, and $b^{\prime \prime}=-\sum b_{n}^{\prime \prime}$. Just as before, if $b^{\prime \prime} \neq 0$ the proof is complete. If $b^{\prime \prime}=0$ we repeat the process once more.

We claim that eventually there must be an integer $p$ for which $b^{(p)} \neq 0$. For if not then

$$
\sum \frac{a_{n}}{\left(\alpha_{n}-\alpha\right)^{p}}=0 \quad(p=0,1,2, \cdots)
$$

Let

$$
g(z)=\sum \frac{a_{n}}{\left(\alpha_{n}-z\right)}, \quad z \in G, z \in\left\{\alpha_{n}\right\} .
$$

From (13) $g$ and all its derivatives must vanish at $\alpha$, so $g \equiv 0$. But this implies that all coefficients vanish, in contradiction to (1). Indeed, if, say, $a_{1} \neq 0$, then by choosing $z$ sufficiently close to $\alpha_{1}, g$ could not vanish. This completes the proof.

This lemma can be somewhat modified. For example, the proof would still work if the $\alpha_{n}$ had at most a countable number of interior limit points, and if $\alpha \notin\left\{\alpha_{n}\right\}$-. This modified lemma would serve equally well for Theorem 3. The proof of the next theorem will suggest additional ways in which the hypothesis of Lemma 4 could be modified; this proof is in Levin's book [11, Chapter 1, §20].

THEOREM 2 (LEVIN). Equation (1) is impossible if there is a compact convex set $D$ containing all the exponents such that at least one exponent $\alpha_{p}$, with $a$ nonzero coefficient $a_{p}$, lies on the boundary of $D$.

Proof. We pass to the Borel transform so that (1) becomes (2), valid for all $D$ in the exterior of $D$. There is an index $N$ such that $\sum_{N+1}^{\infty}\left|a_{n}\right|<1 / 2$. Further, we take $p=1$ and $a_{1}=1$. Then from (2) we have

$$
0 \geqq \frac{1}{\left|z-\alpha_{1}\right|}-\sum_{2}^{N} \frac{\left|a_{n}\right|}{\left|z-\alpha_{n}\right|}-\sum_{N+1}^{\infty} \frac{\left|a_{n}\right|}{\left|z-\alpha_{n}\right|} .
$$


Let $L$ be a line of support to $D$ at $\alpha_{1}$ and let $L^{\prime}$ be the ray perpendicular to $L$, starting at $\alpha_{1}$ and going out away from $D$. If $z \in L^{\prime}$ then $\left|z-\alpha_{1}\right|$ $<\left|z-\alpha_{n}\right|(n=2,3, \cdots)$. Hence

$$
0>\frac{1}{2\left|z-\alpha_{1}\right|}-\sum_{2}^{N} \frac{\left|a_{n}\right|}{\left|z-\alpha_{n}\right|} .
$$

But if $z \rightarrow \alpha_{1}$ on $L^{\prime}$ we have a contradiction and the theorem is proven.

3. The principal results. Let $G$ be a bounded domain. By $E(G)$ we denote the family of all those entire functions $h(z)$ that admit a representation of the form

$$
h(z)=\sum a_{n} \exp \left(\alpha_{n} z\right), \quad \sum\left|a_{n}\right|<\infty, \quad \alpha_{n} \in G .
$$

In case $G$ is the unit circle we shall write simply $E$.

Different domains may have the same sets $E(G)$. It will follow from Theorem 3 that if $G_{1}$ is the unit circle, $G_{2}$ is the unit circle with the unit interval $(0 \leqq x \leqq 1)$ deleted, and $G_{3}$ is any annulus $(r<|z|<1)$, then $E\left(G_{1}\right)=E\left(G_{2}\right)$ $=E\left(G_{3}\right)$.

Clearly $E(G)$ is a linear vector space over the complex numbers. We introduce a norm by the formula

$$
\|h\|=\inf \sum\left|a_{n}\right|
$$

taken over all representations (14) of $h$. We shall see later $(\S 4)$ that if $G$ is a Jordan domain then $E(G)$ is complete in this norm.

As usual, $l_{1}$ and $l_{\infty}$ denote the Banach spaces of absolutely convergent sequences, and of bounded sequences, respectively.

THEOREM 3. Let $G$ be a Jordan region and let $S=\left\{\alpha_{n}\right\}(n=1,2, \cdots)$ be a sequence of distinct points in $G$ with no interior limit points. Then the following three properties of $S$ are equivalent.

(i) $S$ represents zero, i.e., coefficients $\left\{a_{n}\right\} \in l_{1}$ exist, not all zero, such that (1) holds.

(ii) $S$ represents all of $E(G)$, i.e., if $h \in E(G)$ and $\epsilon>0$ are given, then coefficients $\left\{a_{n}\right\}$ exist such that

$$
h(z)=\sum a_{n} \exp \left(\alpha_{n} z\right), \quad \sum\left|a_{n}\right|<\|h\|+\epsilon .
$$

(iii) $\sup _{n}\left|f\left(\alpha_{n}\right)\right|=\|f\|$ for all $f \in H_{\infty}(G)$.

In case $G$ is the unit circle, then these three properties are all equivalent to the following condition.

(iv) Almost every boundary point $p=\exp (i \theta)$ may be approached nontangentially (i.e., inside of some angle with vertex at $p$ ) by points of $S$.

Condition (iii) was suggested by P. C. Rosenbloom, and condition (iv) by G. Piranian.

Proof. The order of proof will be: (iv) $\rightarrow$ (iii) $\rightarrow$ (ii) $\rightarrow$ (i) $\rightarrow$ (iii) $\rightarrow$ (iv). We do not know of any direct way to go from (i) to (ii). 
(iv) $\rightarrow$ (iii). Let $f \in H_{\infty}$ and let $\epsilon>0$ be given. There is a set of positive measure on the boundary of the unit circle such that $\left|f\left(e^{i \theta}\right)\right| \geqq\|f\|-\epsilon$ for $\exp (i \theta)$ in this set. At almost all of these points $f(z)$ has a nontangential limit, and almost all of them are approachable nontangentially by points of $S$; therefore, sup $\left|f\left(\alpha_{n}\right)\right| \geqq\|f\|-\epsilon$.

(iii) $\rightarrow$ (ii). It will be sufficient to show that $S$ represents all exponentials. In other words, we must show that if $\alpha_{0} \in G$ is given $\left(\alpha_{0} \neq \alpha_{n}, n=1,2, \cdots\right)$, and $\epsilon>0$ is given, then there exist coefficients $\left\{a_{n}\right\}$ such that

$$
\exp \left(\alpha_{0} z\right)=\sum_{1}^{\infty} a_{n} \exp \left(\alpha_{n} z\right), \quad \sum_{1}^{\infty}\left|a_{n}\right|<1+\epsilon .
$$

Consider the mapping $T: H_{\infty}(G) \rightarrow l_{\infty}$ defined by $T f=\left\{f\left(\alpha_{n}\right)\right\}_{0}^{\infty}$. By (iii) this is an isometric imbedding of $H_{\infty}(G)$ in $l_{\infty}$. Let $B=T\left(H_{\infty}(G)\right)$. Then $B$ is a norm closed subspace of $l_{\infty}$. Our first goal is to show that $B$ is actually weakstar closed. See Banach [1, Chapitre VIII, and Annexe] and Hille-Phillips $[9, \S 2.10]$ for the relevant definitions and theorems.

Since $l_{\infty}$ is the conjugate space of a separable space, it is sufficient to show that every weak-star convergent sequence in $B$ converges to an element of $B$ [1, Théorème 5 , Chapitre VIII]. Weak-star convergence of a sequence in $l_{\infty}$ is equivalent to boundedness of the norms plus convergence in each fixed coordinate. Since $B$ and $H_{\infty}(G)$ may be identified, a weak-star convergent sequence may be identified with a uniformly bounded sequence of functions $\left\{f_{k}\right\} \subset H_{\infty}(G)$ such that $\lim f_{k}\left(\alpha_{n}\right)(k \rightarrow \infty)$ exists for each fixed $n$.

The functions $\left\{f_{k}\right\}$ form a normal family. There cannot be two subsequences converging to different limits $f$ and $g$, for $f$ and $g$ would agree at the points $\alpha_{n}$ and then by (iii) $f-g$ would vanish identically. Therefore $g(z)$ $=\lim f_{k}(z)$ exists, uniformly on compact subsets of $G$. Thus $g$ is the weakstar limit of the sequence $\left\{f_{k}\right\}$, and therefore $B$ is weak-star closed.

Consider now the element $p=(1,0,0, \cdots) \in l_{\infty}$. We show that $\operatorname{dist}(p, B)$ $=1 / 2$. Indeed, the constant function $f \equiv 1 / 2$ has distance $1 / 2$ from $p$, and if any $f \in H_{\infty}(G)$ were at a smaller distance from $p$ then we would have $\left|f\left(\alpha_{o}\right)\right|>\sup \left|f\left(\alpha_{n}\right)\right|(n \geqq 1)$, contrary to (iii).

Now we require a theorem of Banach which states that if $E$ is a Banach space, $E^{*}$ its conjugate, $B$ a weak-star closed subspace of $E^{*}, p \notin B, \epsilon>0$, then there exists an $x \in E$ such that: $x \perp B,(x, p)=1,\|x\|<(1 / d)+\epsilon$, where $d=\operatorname{dist}(p, B)$ and $(x, p)$ denotes the value of the linear functional $p$ at the point $x$.

Applying this lemma to our present situation we obtain $\left\{a_{n}\right\}_{0}^{\infty} \in l_{1}$ such that:

I. $\sum_{0}^{\infty} a_{n} f\left(\alpha_{n}\right)=0$ for all $f \in H_{\infty}(G)$;

II. $a_{\mathrm{o}}=1$;

III. $\sum_{0}^{\infty}\left|a_{n}\right|<2+\epsilon$. 
By Lemma 3 we see that I is equivalent to $\sum a_{n} \exp \left(\alpha_{n} z\right) \equiv 0$. Now applying II and III we have (17), except for a change of sign in the coefficients. This completes the proof of (ii).

(ii) $\rightarrow$ (i). Let $\alpha_{o}$ be any point in $G$ different from all $\alpha_{n}$. By (ii) there exist coefficients such that (17) holds. If we apply Lemma 2 to (17) with $f(w)$ $=w-\alpha_{0}$ we obtain (i).

(i) $\rightarrow$ (iii). Assume that (i) holds but that (iii) does not. Then there is an $f \in H_{\infty}(G)$ and an $\alpha(|\alpha|<1)$ for which $|f(\alpha)|>\sup \left|f\left(\alpha_{n}\right)\right|$. By Lemma 4 there exist coefficients such that

$$
\exp (\alpha z)+\sum b_{n} \exp \left(\alpha_{n} z\right) \equiv 0, \quad \sum\left|b_{n}\right|<\infty .
$$

Without loss of generality we may assume that $f(\alpha)=1$. Then by Lemma 3 for each positive integer $p$ we have $1+\sum b_{n}\left[f\left(\alpha_{n}\right)\right]^{p}=0$. But this is impossible for large $p$.

(iii) $\rightarrow$ (iv). Let us assume that condition (iv) fails; we shall show that then condition (iii) also fails. Since (iv) is false, there is a set $E$ of positive measure on the unit circumference $|z|=1$, such that no point of $E$ can be approached nontangentially by the $\alpha_{n}$. This means that any angle with vertex at a point of $E$ can contain only a finite number of the $\alpha_{n}$. In particular this is true for a right angle, placed so that the radius to the point bisects the angle.

This implies that at each point $p=\exp (i \theta) \in E$ there is a right triangle $\triangle_{\theta}$, with the right angle vertex at the point $p$ and the other two vertices inside the unit circle, having the radius to $p$ as an axis of symmetry, and containing none of the $\alpha_{n}$. There will be a number $b>0$ and a closed subset $E_{1} \subset E$ of positive measure such that at each point of $E_{1}$ the altitude of the triangle $\triangle_{\theta}$, measured from the vertex $p$, has length $\geqq b$.

Choose now a closed arc $I$, whose endpoints are in $E_{1}$, for which $\left|E_{1} \cap I\right|$ $>0$ and $|I|<b$, where the vertical bars denote Lebesgue measure. Let $G$ be the complement of $E_{1}$ with respect to the $\operatorname{arc} I$; then $G$ is the union of a set of open $\operatorname{arcs}\left\{I_{n}\right\}$. (If $I \subset E_{1}$, then $G$ is the null set.) Take one of these arcs, $I_{j}$, with endpoints $\exp (i \alpha)$ and $\exp (i \beta)$, and draw the two triangles $\triangle_{\alpha}$ and $\triangle_{\beta}$. Then one sees easily that the sides of these triangles cross over the interval $I_{j}$ to form a little "triangle" $T_{j}$, one side of which is the $\operatorname{arc} I_{j}$. Thus if $t$ denotes a point of $I_{j}$, then any of the $\alpha_{n}$ sufficiently near to $t$ must lie in $T_{j}$.

Let $k(\phi)$ be the characteristic function of the set $G$, and define $f(z)$ by

$$
f(z)=\exp \left\{\frac{1}{2 \pi} \int_{0}^{2 \pi} k(\phi) \frac{z+e^{i \phi}}{z-e^{i \phi}} d \phi\right\} .
$$

Then

$$
|f(z)|=\exp \left\{\frac{-1}{2 \pi} \int_{0}^{2 \pi} k(\phi) \frac{1-r^{2}}{\left|z-e^{i \phi}\right|^{2}} d \phi\right\}
$$


for $z=r \exp (i \theta)$, and so $|f(z)| \leqq 1$ for $|z|<1$.

It is a well known property of the Poisson integral that

$$
\lim \left|f\left(r e^{i \theta}\right)\right|=1
$$

at almost all points $\exp (i \theta)$ of $E_{1}$.

We shall now show that

$$
|f(z)| \leqq e^{-1 / 2} \quad\left(z \in T_{j}, j=1,2, \cdots\right) .
$$

Consider the $\operatorname{arc} I_{j}$, with endpoints $\exp (i \alpha)$ and $\exp (i \beta) \quad(0 \leqq \alpha \leqq \beta<2 \pi)$. From (19) we have $|f(z)|=\prod\left|f_{n}(z)\right|$ where

$$
f_{n}(z)=\exp \left\{\frac{1}{2 \pi} \int_{I_{n}} \frac{z+e^{i \phi}}{z-e^{i \phi}} d \phi\right\} .
$$

Since each $\left|f_{n}(z)\right| \leqq 1$ for $|z|<1$ this means that $|f(z)| \leqq\left|f_{j}(z)\right|$. So (21) will be proven if we can show that

$$
\frac{1}{2 \pi} \int_{\alpha}^{\beta} \frac{1-|z|^{2}}{\left|z-e^{i \phi}\right|^{2}} d \phi \geqq \frac{1}{2}
$$

It is well known that this integral has a simple geometrical interpretation (see, for example, Nevanlinna [5, pp. 6-7]). Namely, one extends the line segment from $\exp (i \alpha)$ to $z$ till it meets the boundary of the unit circle at a point $w_{1}$. Similarly, extend the line segment from $\exp (i \beta)$ to $z$ till it meets the boundary in a point $w_{2}$. Then the integral is equal to the arc length from $w_{1}$ to $w_{2}$ (in the counterclockwise direction) divided by $2 \pi$. Using this, one sees that the minimum of the integral in (22) for $z \in T_{j}$ is attained at the interior vertex of $T_{j}$, and that this minimum value is $1 / 2+(\beta-\alpha) / 2 \pi$. This establishes (22) and therefore also (21).

Now let $t=\exp (i \theta)$ be a point of $E_{1}$, interior to the arc $I$, at which the relation (20) holds. For simplicity we assume $t=1$. Then there is an $\epsilon>0$ such that if $\operatorname{Re}\left(\alpha_{n}\right)>1-\epsilon$, then $\alpha_{n}$ is in one of the "triangles" $T_{j}$. By (21), $\left|f\left(\alpha_{n}\right)\right|$ $\leqq \exp (-1 / 2)$ at all such points $\alpha_{n}$. Let $g(z)=f(z) e^{z}$. Then $g \in H_{\infty}$ and $\|g\|=e$. But

$$
\begin{array}{ll}
\left|g\left(\alpha_{n}\right)\right| \leqq e^{1 / 2} & \text { for } \operatorname{Re}\left(\alpha_{n}\right)>1-\epsilon, \\
\left|g\left(\alpha_{n}\right)\right| \leqq e^{1-\epsilon} & \text { for } \operatorname{Re}\left(\alpha_{n}\right) \leqq 1-\epsilon,
\end{array}
$$

and so $\sup \left|g\left(\alpha_{n}\right)\right|<\|g\|$. In other words, condition (iii) is not satisfied.

This completes the proof of Theorem 3.

REMARK 1 . The assumption that the $\alpha_{n}$ have no interior limit points in $G$ was only needed for the implication (i) $\rightarrow$ (iii) where Lemma 4 was applied. As noted after Lemma 4 , this assumption can be weakened slightly.

REMARK 2. Let $\left\{\beta_{n}\right\}$ be a sequence of points inside the unit circle for which $\sum\left(1-\left|\beta_{n}\right|\right)<\infty$. Then almost no boundary points are approachable nontangentially by subsequences of $\left\{\beta_{n}\right\}$. 
Indeed, let $B$ be the set of boundary points that can be approached nontangentially. Let $f \in H_{\infty}(f \not \equiv 0)$ vanish on $\left\{\beta_{n}\right\}$. Then $f$ has radial limits almost everywhere on $B$, and these limits must all be zero as the radial limit is the same as the limit inside of any angle. But the limit cannot be zero on a set of positive measure, and therefore $|B|=0$.

The converse to this remark is false. In fact, one can give examples of sequences $\left\{\beta_{n}\right\}$ such that almost no boundary points are approachable nontangentially, and such that if $C$ is any sector of the unit circle then

$$
\sum_{\beta_{n} \in C}\left(1-\left|\beta_{n}\right|\right)=\infty \text {. }
$$

REMARK 3. If $\left\{\alpha_{n}\right\}$ satisfies condition (iv) of Theorem 3 and $\left\{\alpha_{n_{j}}\right\}$ is a subsequence such that

$$
\sum\left(1-\left|\alpha_{n_{j}}\right|\right)<\infty
$$

then $\left\{\alpha_{n}\right\} \backslash\left\{\alpha_{n}\right\}$ satisfies condition (iv) (the symbol \denotes set-theoretic difference).

This follows from Remark 2.

REMARK 4. Let $\left\{\alpha_{n}\right\}$ satisfy condition (iv). Then there are coefficients $\left\{a_{n}\right\}$, all of which are different from zero, such that (1) holds. Further, if $h$ is any entire function admitting a representation (14) then the coefficients $\left\{a_{n}\right\}$ may be chosen all different from zero.

Indeed, by (ii) there are coefficients $\left\{a_{i 1}\right\}(i=2,3, \cdots)$ such that

$$
0=\frac{1}{2} \exp \left(\alpha_{1} z\right)+\sum_{i=2}^{\infty} a_{i 1} \exp \left(\alpha_{i} z\right), \quad \sum_{2}^{\infty}\left|a_{i 1}\right|<1 .
$$

Choose $b_{2}$ such that $\left|b_{2}\right|<1 / 4$ and $b_{2} \neq-a_{21}$. Then applying (ii) again we find coefficients $\left\{a_{i 2}\right\}(i=3,4, \cdots)$ such that

$$
0=b_{2} \exp \left(\alpha_{2} z\right)+\sum_{i=3}^{\infty} a_{i 2} \exp \left(\alpha_{i} z\right), \quad \sum_{3}^{\infty}\left|a_{i 2}\right|<\frac{1}{2} .
$$

Choose $b_{3}$ such that $\left|b_{3}\right|<1 / 8$ and $b_{3} \neq-\left(a_{31}+a_{32}\right)$. Then there are coefficients $\left\{a_{i 3}\right\}(i=4,5, \cdots)$ such that

$$
0=b_{3} \exp \left(\alpha_{3} z\right)+\sum_{i=4}^{\infty} a_{i 3} \exp \left(\alpha_{i} z\right), \quad \sum_{4}^{\infty}\left|a_{i 3}\right|<\frac{1}{4} .
$$

This process may be continued. If we add up all the resulting equations we have a representation of zero in which no coefficients vanish.

Let now $h$ admit a representation (14) and let $\left\{b_{n}\right\}$ all different from zero be chosen such that $\sum b_{n} \exp \left(\alpha_{n} z\right) \equiv 0$. Then

$$
h(z)=\sum a_{n} \exp \left(\alpha_{n} z\right)+\lambda \sum b_{n} \exp \left(\alpha_{n} z\right)
$$


for all complex $\lambda$. It only remains to choose $\lambda$ such that

$$
a_{n}+\lambda b_{n} \neq 0 \quad(n=1,2, \cdots) .
$$

REMARK 5. Let $\left\{\alpha_{n}\right\}$ satisfy condition (iv) and let $\left\{\alpha_{n_{j}}\right\}$ be a subsequence for which (23) holds. Let $h(z)$ be any entire function admitting a representation (14), and let $\left\{b_{j}\right\} \in l_{1}$ be given. Then one can find coefficients $\left\{a_{n}\right\} \in l_{1}$ such that $h(z)=\sum a_{n} \exp \left(\alpha_{n} z\right)$ and $a_{n_{j}}=b_{j}$. In other words, certain infinite subsets of coefficients can be prescribed arbitrarily in $l_{1}$.

Indeed, let $\phi(z)=\sum b_{j} \exp \left(\alpha_{n_{j}} z\right)$. By Remark 2 both $\phi(z)$ and $h(z)$ can be represented in terms of the exponents $\left\{\alpha_{n}\right\} \backslash\left\{\alpha_{n_{j}}\right\}$ :

$$
\begin{array}{ll}
\phi(z)=\sum c_{n} \exp \left(\alpha_{n} z\right) & \left(c_{n_{j}}=0, j=1,2, \cdots\right), \\
h(z)=\sum d_{n} \exp \left(\alpha_{n} z\right) & \left(d_{n_{j}}=0, j=1,2, \cdots\right) .
\end{array}
$$

Then

$$
h(z)=\sum d_{n} \exp \left(\alpha_{n} z\right)-\sum c_{n} \exp \left(\alpha_{n} z\right)+\sum b_{j} \exp \left(\alpha_{n j} z\right),
$$

so we may choose $a_{n}=\left(d_{n}-c_{n}\right)$ for $n \in\left\{n_{j}\right\}$, and $a_{n_{j}}=b_{j}$.

REMARK 6. It is easy to give examples of sequences $\left\{\alpha_{n}\right\}$ satisfying condition (iv). For example, let $r_{n} \rightarrow 1\left(r_{n}<1\right)$, let $c>0$ be given, and let $\phi(n)$ be any integer-valued function for which

$$
\phi(n)>\frac{c}{1-r_{n}} \quad(n=1,2, \cdots) .
$$

The sequence $\left\{\alpha_{n}\right\}$ is now formed by taking $\phi(n)$ points, equally spaced, on the circle $|z|=r_{n}(n=1,2, \cdots)$. It can be verified that every boundary point $p$ can be approached inside of an angle of opening $2 \beta$, placed symmetrically about the radius to $p$, for all $\beta$ such that $\tan \beta>\pi / c$.

From this it follows by conformal mapping that in any simply connected domain $G$ (with at least two boundary points) sequences $\left\{\alpha_{n}\right\}$ exist that satisfy (iii), and have no interior limit points.

REMARK 7. It might be asked whether one couldn't require something more than absolute convergence of the coefficients in (1). For example, is it possible to have a representation of zero in which $\sum\left|a_{n}\right|^{\delta}<\infty$ for every $\delta>0$ ?

In this connection Denjoy has shown that if $D$ is any closed set in the extended plane, and $U$ is an open set containing $D$, and $\phi$ is any function analytic on $U$ (and vanishing at $z=\infty$ if $D$ is unbounded), and $\epsilon>0$ is given, then there exist sequences $\left\{a_{n}\right\},\left\{\alpha_{n}\right\}$, with $\alpha_{n} \in D(n=1,2, \cdots)$ such that

$$
\phi(z)=\sum \frac{a_{n}}{\alpha_{n}-z}
$$

and

$$
\left|a_{n}\right|<k \exp \left(-n^{1 / 2-e}\right) .
$$


See $[5$, p. 3] and [6].

Remark 8. The sets $\left\{\alpha_{n}\right\}$ that satisfy condition (iv) seem to play a role analogous to the boundary. Thus one is led to look for analogues of theorems about the boundary. Consider, for example, the following theorem: if $f$ is analytic for $|z|<1$ and continuous for $|z| \leqq 1$ and if $f$ is schlicht on the boundary, then $f$ is schlicht inside.

An analogue might be: let $\left\{\alpha_{n}\right\}$ satisfy condition (iv) and let $f \in H_{\infty}$ be such that to every $\delta>0$ there corresponds an $\epsilon>0$ such that $\left|\alpha_{n}-\alpha_{m}\right| \geqq \delta$ implies $\left|f\left(\alpha_{n}\right)-f\left(\alpha_{m}\right)\right| \geqq \epsilon$; then $f$ is schlicht in $|z|<1$. (It would not be enough to require merely that $f$ be one-to-one on $\left\{\alpha_{n}\right\}$; for example, if no two of the $\alpha_{n}$ are diametrically opposed then $z^{2}$ is one-to-one on $\left\{\alpha_{n}\right\}$.) We do not know if this conjecture is true, nor do we know if this property implies condition (iv).

Consider now the problem of determining the values of an analytic function inside the circle by means of its values on or near the boundary. For example, if $f$ is analytic for $|z|<1$ and continuous for $|z| \leqq 1$ then the Cauchy integral

$$
f(z)=\frac{1}{2 \pi i} \int_{|w|-1} f(w) \frac{1}{w-z} d w
$$

gives the solution to the problem. This may be written

$$
f(z)=\int f(w) d \mu_{z}(w)
$$

where the measure $\mu_{z}$ depends only on the point $z$ and not on the function $f$. Another possible choice for the measures $\mu_{z}$ is

$$
d \mu_{z}(w)=\left(\frac{1}{w-z}+\frac{\bar{z}}{1-\bar{z} w}\right) d w
$$

(Macintyre, Rogosinski [12, p. 304]). These measures are all of total variation 1 on $|w|=1$, independently of $z(|z|<1)$. (The absolute value of the expression in parentheses is the Poisson kernel.)

Theorem 3 enables us to obtain a formula analogous to (24), with the integral replaced by a series. Again let $S=\left\{\alpha_{n}\right\}$ be a sequence of distinct points inside the unit circle with no interior limit points.

THEOREM 4. The sequence $S=\left\{\alpha_{n}\right\}$ has the property (v) defined below if and only if it has property (i) of Theorem 3.

(v) For each point $z$ inside the unit circle and each $\epsilon>0$ there exist coefficients $\left\{a_{n}(z ; \epsilon)\right\}$ for which

$$
f(z)=\sum a_{n}(z ; \epsilon) f\left(\alpha_{n}\right), \quad \sum\left|a_{n}(z ; \epsilon)\right|<1+\epsilon,
$$

for all $f \in H_{\infty}$. 
Proof. Let (v) hold, and choose a point $z_{\mathrm{o}} \in S$. From (26) and Lemma 3 we obtain a representation of zero using $z_{\mathrm{o}}$ and $\left\{\alpha_{n}\right\}$. Using Lemma 2 we can drop out the $z_{0}$ term, thus obtaining (1).

Conversely, let (1) hold, and assume $z_{\mathrm{o}} \notin S$ (since otherwise (26) is trivial). Then (26) follows from (17) and Lemma 3.

Unfortunately, Theorem 4 does not give any explicit method for determining the coefficients $\left\{a_{n}(z ; \epsilon)\right\}$. If one seeks to eliminate the $\epsilon$ in (v), then one must eliminate the $\epsilon$ in (17). We do not know whether this can be done or not. However, J. Wolff [21] has shown that if $z_{0}$ is given, then there exist numbers $\left\{\alpha_{n}\right\}$ with $z_{0} \notin\left\{\alpha_{n}\right\}$, and there exist coefficients $\left\{a_{n}\right\}$ such that (26) holds with $\epsilon=0$. In his example the $\alpha_{n}$ have many interior limit points. See the remarks following Theorem 8 of $\$ 4$ for a further discussion.

We now mention two applications of Theorem 3 that were pointed out to us by John Wermer. Let $\left\{\alpha_{n}\right\}$ be a bounded sequence of distinct complex numbers. We shall say that $\left\{\alpha_{n}\right\}$ "represents zero" if coefficients $\left\{a_{n}\right\}$ can be found, not all zero, such that (1) holds.

Let $T$ be a bounded normal operator on Hilbert space $H$. Let the eigenvectors of $T$ span $H$ and let $\alpha_{1}, \alpha_{2}, \cdots$ be the distinct eigenvalues of $T$. Wermer [20] has shown that the following statements are equivalent.

(a) Every invariant subspace of $T$ is spanned by the eigenvectors it contains.

(b) Every invariant subspace of $T$ contains an eigenvector.

(c) Every subspace invariant under $T$ is also invariant under $T^{*}$.

(d) $\left\{\alpha_{n}\right\}$ does not represent zero.

J. E. Scroggs [17] has a number of further results in this direction.

Tord Hall [7] has considered the following problem. Let $\left\{A_{n}\right\},\left\{\alpha_{n}\right\}$ be two sequences of complex numbers with the $\alpha_{n}$ all distinct and different from zero. It is further assumed that $A_{n} \geqq 1(n=1,2, \cdots)$ and that

$$
\lim _{n}\left|\alpha_{n}\right|^{k} / A_{n}=0
$$

Let $Q$ denote the set of all polynomials such that $\left|p\left(\alpha_{n}\right)\right| \leqq A_{n}(n=1,2, \cdots)$, and let $M=\sup |p(0)|(p \in Q)$.

Hall proves that $M<\infty$ if and only if there exist coefficients $\left\{a_{n}\right\}$ such that

$$
\sum\left|a_{n}\right| \leqq 1, \quad \sum_{n} \frac{a_{n}}{A_{n}}\left(\alpha_{n}\right)^{k}=0(k>0), \quad \sum \frac{\alpha_{n}}{A_{n}} \neq 0 .
$$

Condition (27) with $k=0$ implies $A_{n} \rightarrow \infty$. If the set $\left\{\alpha_{n}\right\}$ is bounded, then condition (28) is equivalent to

$$
\sum \frac{a_{n}}{A_{n}} \exp \left(\alpha_{n} z\right) \equiv \text { const. }(\neq 0), \quad \sum\left|a_{n}\right| \leqq 1 .
$$


Thus if $\left\{\alpha_{n}\right\}$ cannot represent zero, then $M=\infty$, no matter how the $\left\{A_{n}\right\}$ are chosen.

4. Functions representable by exponentials. In this section we study the space $E(G)$ where $G$ is a Jordan region (see (14)).

THEOREM 5. Let $G$ be a Jordan region. Then $E(G)$ is a Banach space in the norm (15), and the conjugate space is $H_{\infty}(G)$ with the supremum norm.

Proof. By Theorem 3 and Remark 6 there exists a sequence $\left\{\alpha_{n}\right\}$ in $G$ such that every function in $E(G)$ can be represented using only the exponents $\left\{\alpha_{n}\right\}$, and the norm is not increased. Let $N$ denote the subset of $l_{1}$ consisting of those sequences $\left\{a_{n}\right\}$ for which $\sum a_{n} \exp \left(\alpha_{n} z\right) \equiv 0 . N$ is a closed linear subspace of $l_{1}$ and $E(G)$ is isomorphic to the quotient space $l_{1} / N$. Hence $E(G)$ is a Banach space.

The conjugate space of $l_{1} / N$ is $N^{\perp}$, the set of all elements in $l_{\infty}$ that are orthogonal to $N$. Let $B$ be the set of those bounded sequences that come from bounded analytic functions: $\left\{f\left(\alpha_{n}\right)\right\}, f \in H_{\infty}(G)$. One half of Lemma 3 tells us that $B \subset N^{\perp}$, while the other half tells us that $B$ is weak-star dense in $N^{\perp}$ (since $\left.N^{\perp \perp}=N\right)$. The proof of (iii) $\rightarrow$ (ii) in Theorem 3 showed that $B$ is weak-star closed. Hence $B=N^{\perp}$. Since $\|f\|=\sup \left|f\left(\alpha_{n}\right)\right|$, the conjugate space is isomorphic to $H_{\infty}(G)$, which completes the proof.

Let $h=\sum a_{n} \exp \left(\alpha_{n} z\right) \in E(G)$, and let $f \in H_{\infty}$. Then $(h, f)$, the value of the linear functional $f$ at the point $h$, is given by

$$
(h, f)=\sum a_{n} f\left(\alpha_{n}\right) .
$$

If $G$ is the unit circle this can be rewritten in terms of the Taylor series of $f$ and $h$. Let $h=\sum A_{n} z^{n} / n$ ! and $f=\sum B_{n} z^{n}$. It can be shown that

$$
(h, f)=\lim _{r \rightarrow 1^{-}} \sum A_{n} B_{n} r^{n}
$$

(first consider the case when the Taylor series for $f$ contains but a single term).

Let now $G$ be the unit circle. By $H_{1}$ we denote as usual the Hardy space of functions $f(z)$, analytic in the unit circle and satisfying the condition

$$
\|f\|_{1}=\lim _{r \rightarrow 1^{-}} \frac{1}{2 \pi} \int_{0}^{2 \pi}\left|f\left(r e^{i \theta}\right)\right| d \theta<\infty .
$$

$H_{1}$ is a Banach space in this norm (whose conjugate space is not $H_{\infty}$ !). The radial limits $f\left(e^{i t}\right)=\lim f\left(r e^{i t}\right)\left(r \rightarrow 1^{-}\right)$exist for almost all $t$, and $f\left(e^{i t}\right) \in L_{1}(0,2 \pi)$. Further, $\left\|f\left(e^{i t}\right)\right\|_{L_{1}}=\|f\|_{H_{1}}$, so $H_{1}$ is isometrically imbedded as a subspace of $L_{1}$.

Let $F$ denote the Banach space $L_{1} / H_{1} . F$ can be interpreted as the space of those entire functions $h(z)$ that admit a representation

$$
h(z)=\frac{1}{2 \pi i} \int_{|w|=1} e^{z w} \phi(w) d w \quad\left(\phi \in L_{1}(0,2 \pi)\right) .
$$


(We shall say that $\phi$ represents $h$.)

Indeed, if $\int e^{z w} \phi(w) d w \equiv 0$ then the Fourier coefficients of $\phi$ vanish on one side and therefore $\phi \in H_{1}$ [15, Chapter 2]. Conversely, if $\phi \in H_{1}$ then $\int e^{z w} \phi(w) d w \equiv 0$ by the Cauchy integral theorem.

The norm in $F$ is the quotient space norm:

$$
\|h\|_{F}=\inf \|\phi\|_{L_{1}}
$$

taken over all $\phi$ that represent $h$.

The conjugate space of $F$ is the annihilator of $H_{1}$ in $L_{\infty}$. If $f \in L_{\infty}, \phi \in L_{1}$ we denote the value of the functional $f$ at the point $\phi$ by $(\phi, f)$, defined by

$$
(\phi, f)=\frac{1}{2 \pi i} \int_{|w|=1} f(w) \phi(w) d w .
$$

If $\phi \in H_{1}$ then it is analytic inside the unit circle. If $f$ is the boundary function of a function in $H_{\infty}$ then $(\phi, f)=0$ by the Cauchy integral theorem. Conversely, if $(\phi, f)=0$ for all $\phi \in H_{1}$ then the Fourier coefficients of $f$ vanish on one side and therefore $f \in H_{\infty}$.

Thus the conjugate space of $F$ is $H_{\infty}$ with the supremum norm. If $h(z)$ $=\sum A_{n} z^{n} / n ! \in F$ and $f(z)=\sum B_{n} z^{n} \in H_{\infty}$ then

$$
(h, f)=\lim _{r \rightarrow 1^{-}} \sum A_{n} B_{n} r^{n} .
$$

THEOREM 6. The two function spaces $E$ and $F$ with the norms (15) and (33) are identical.

Proof. We first show that $F$ contains all pure exponentials $e^{\alpha z}(|\alpha|<1)$, and that $\left\|e^{\alpha z}\right\|_{F} \leqq 1$. Indeed, let

$$
K_{\alpha}(w)=\frac{1}{w-\alpha}+\frac{\bar{\alpha}}{1-\bar{\alpha} w}
$$

(see (25)). Then $e^{\alpha z}$ is represented by $K_{\alpha}(w)$ and $\left\|K_{\alpha}\right\|=1$, which is the result.

It follows that $E \subset F$ since by definition $E$ consists of all absolutely convergent series of exponentials. Also, $E$ and $F$ have the same conjugate space: $H_{\infty}$ in the supremum norm. If $h \in E$ and $f \in H_{\infty}$ then the value of the linear functional $f$ at the point $h,(h, f)$, is the same whether we regard $h$ as an element of $E$ or as an element of $F$ (see (30) and (35)).

Therefore $\|h\|_{E}=\|h\|_{F}$ for all $h \in E$, since $\|h\|=\sup |(h, f)|$, taken over all $f \in H_{\infty}$ of norm one. So $E$ is a closed subspace of $F$. But it cannot be a proper subspace, for then there would be a linear functional vanishing on $E$ but not vanishing identically, which is impossible.

The first corollary is due to Wolff [21].

Corollary 1. E contains all entire functions of exponential type less than one. 
Proof. For such a function the Borel transform is analytic part way inside the unit circle, and in the Borel transform representation of $h$ (see (4)) we may take $r=1$. $F(w)$ is analytic on $|w|=1$, and thus is in $L_{1}(0,2 \pi)$.

In fact $E$ contains all those entire functions whose Borel transforms are in $H_{1}$ in the exterior of the unit circle.

Definition. A sequence $\left\{A_{n}\right\}(n=0,1, \cdots)$ will be said to be a Fourier sequence on one side if numbers $A_{-1}, A_{-2}, \cdots$ exist such that $\left\{A_{n}\right\}$ $(-\infty<n<\infty)$ is the sequence of complex Fourier coefficients of some integrable function on $(0,2 \pi)$.

Corollary 2. A necessary and sufficient condition that $\left\{A_{k}\right\}(k=0,1, \cdots)$ be a Fourier sequence on one side is that there exist two sequences of complex numbers, $\left\{a_{n}\right\},\left\{\alpha_{n}\right\}\left(\sum\left|a_{n}\right|<\infty,\left|\alpha_{n}\right|<1(n=1,2, \cdots)\right)$ such that

$$
\sum_{n=1}^{\infty} a_{n}\left(\alpha_{n}\right)^{k}=A_{k} \quad(k=0,1,2, \cdots) .
$$

Proof. Let $\left\{a_{n}\right\},\left\{\alpha_{n}\right\}$ be given and let $h(z)=\sum a_{n} \exp \left(\alpha_{n} z\right)$. By Theorem 6 there is a $\phi \in L_{1}$ such that $h(z)=(1 / 2 \pi i) \int e^{z w} \phi(w) d w$. Differentiating these two representations $k$-times and evaluating at $z=0$ we obtain (37), where the $A_{k}$ are the Fourier coefficients on one side of $\phi$.

Conversely, given $\phi \in L_{1}$ we define $h$ by (32). By Theorem $4, h \in E$ and so $h$ also has a representation (14). Just as in the preceding paragraph we obtain (37). In other words, the coefficients of any given $\phi \in L_{1}$ have a representation (37).

REMARK 9. If $\mu$ is a measure concentrated entirely on the unit circumference $|w|=1$, and $\mu$ is not purely absolutely continuous, then $\int e^{z w} d \mu(w)$ is not in $E$.

Indeed, if it were in $E$ then by Theorem 4 there would be a purely absolutely continuous measure $\sigma$ on $|w|=1$ such that $\int e^{z w} d(\mu-\sigma) \equiv 0$. But by a theorem of F. and M. Riesz [15, Chapter 2, §5] this would imply that $\mu-\sigma$ were absolutely continuous, which would be a contradiction.

H. S. Shapiro has pointed out to us that the work of Macintyre-Rogosinski [12], Havinson [8], and Rogosinski-Shapiro [16] provides further information about the space $E$ and enables us to compute the norms of elements in $E$ in simple cases. (There is considerable duplication in the last two papers, the last one having been written in ignorance of Havinson's work.)

Theorem A of [16] applied to $E$ yields the following result.

THEOREM 7. For each $h \in E(h \neq 0)$ there exists a unique $\phi \in L_{1}$ and a unique $f \in H_{\infty}$ such that $\phi$ represents $h\left(\right.$ see (32)) and $\|h\|_{E}=\|\phi\|_{L_{1}}=(h, f)$.

Thus the infimum in (33) is uniquely attained.

Corollary. Let $h \in E$. Then $\left\|h^{\prime}\right\| \leqq\|h\|$, and there is a unique $h_{1} \in E$ such that $h_{1}^{\prime}=h$ and $\left\|h_{1}\right\|=\|h\|$. 
Proof. The first statement is obvious since $\sum\left|a_{n} \alpha_{n}\right|<\sum\left|a_{n}\right|$. For the second statement, let $\phi \in L_{1}$ provide the minimal representation of $h$ as in Theorem 7. Then $\phi(w) / w=\phi_{1}(w)$ represents $h_{1}$, and $\left\|\phi_{1}\right\|=\|\phi\|$. There could not be a second such primitive, say $h_{2}$, represented minimally by some $\phi_{2}$. For then $w \phi_{2}$ would represent $h_{2}^{\prime}=h$, but the minimal representation of $h$ is unique.

REMARK 10. If $h \in E$ then $|h(0)| \leqq\|h\|$. Indeed, $|h(0)|=\left|\sum a_{n}\right| \leqq \sum\left|a_{n}\right|$.

We now give the norms of several elements of $E$, and of one linear operator on $E$.

1. $\left\|e^{\alpha z}\right\|=1(|\alpha|<1)$.

2. $\left\|z^{n} / n !\right\|=1(n=0,1,2, \cdots)$.

3. $\left\|z e^{\alpha z}\right\|=1 /\left(1-|\alpha|^{2}\right)$.

4. ||$e^{\alpha z}-1 \|=2|\alpha| /\left(1+\left(1-|\alpha|^{2}\right)^{1 / 2}\right)$

5. $\left\|e^{b z}-e^{a z}\right\|=2|c| /\left(1+\left(1-|c|^{2}\right)^{1 / 2}\right) \quad(c=(b-a) /(1-\bar{a} b))$.

6. $\left\|D_{\mathrm{o}}^{-1}\right\|=2$, where $D_{\mathrm{o}}^{-1}$ is the integration operator $\left(D_{\mathrm{o}}^{-1} h\right)(z)=\int_{0}^{2} h(s) d s$.

Proof. 1. This is obvious from Remark 10 and the definition of norm.

2. $z^{n} / n$ ! can be represented by $1 / w^{n+1}$, and hence $\left\|z^{n} / n !\right\| \leqq 1$. By differentiating $n$ times we get the reverse inequality.

3. Since $\|h\|=\sup |(h, f)|(\|f\| \leqq 1)$, the assertion to be proven is equivalent to: $\sup \left|f^{\prime}(\alpha)\right|=1 /\left(1-|\alpha|^{2}\right)$. This result is given on page 304 of [12].

4. $\left\|e^{\alpha z}-1\right\|=\sup |f(\alpha)-f(0)|$ taken over all $f$ in the unit ball of $H_{\infty}$. By Theorem 16 of [16] the extremal $f$ has the form: $f(z)=(z-a) /(1-\bar{a} z)$. Therefore,

$$
|f(\alpha)-f(0)|=\frac{|\alpha|\left(1-|a|^{2}\right)}{|1-\bar{a} \alpha|} .
$$

It follows that the extremal $f$ must have $a=r \alpha /|\alpha|$ for some $0<r<1$. Therefore,

$$
|f(\alpha)-f(0)|=\frac{|\alpha|\left(1-r^{2}\right)}{1-r|\alpha|}=\phi(r) .
$$

We wish to maximize $\phi(r)$. Putting the derivative equal to zero and solving for $r$ we obtain: $r|\alpha|=1-\left(1-|\alpha|^{2}\right)^{1 / 2}$. (The plus sign is excluded since $r$ must lie in the unit interval.) Substituting this value for $r$ into the previous equation and simplifying we obtain the result.

5. sup $|f(b)-f(a)|=\sup |f(\phi(b))-f(\phi(a))|$ for any bilinear map $\phi$ of the circle onto itself. Choose $\phi=(z-a) /(1-\bar{a} z)$, which reduces this case to the previous one: $\left\|e^{b z}-e^{a z}\right\|=\left\|e^{c z}-1\right\|$ for $c=(b-a) /(1-\bar{a} b)$.

6 . Let $h \in E$ be given and let $h_{1}$ be the minimal primitive (see the corollary to Theorem 7$)$. Then $\left(D_{0}^{-1} h\right)(z)=h_{1}(z)-h_{1}(0)$, and therefore $\left\|D_{0}^{-1} h\right\|$ $\leqq 2\|h\|$. On the other hand, $\left\|D_{0}^{-1} e^{\alpha z}\right\|=(1 / \alpha)\left\|e^{\alpha z}-1\right\| \rightarrow 2$ as $\alpha \rightarrow 1$, from 5 . 
We now consider the "positive" elements in $E$. Let $E_{+}$be the set of all those $h \in E$ for which

$$
h(0)=\|h\| .
$$

Clearly $E_{+}$is a positive cone, i.e., it is closed under addition and under multiplication by non-negative scalars. Also, if $h_{1}, \cdots, h_{n} \in E_{+}$then $\left\|h_{1}+\cdots+h_{n}\right\|=\left\|h_{1}\right\|+\cdots+\left\|h_{n}\right\|$.

THEOREM 8. $h \in E_{+}$if and only if $h$ can be represented by a $\phi \in L_{1}$ for which $w \phi(w) \geqq 0$ almost everywhere on $|w|=1$. In this case $\phi$ provides the unique minimal representation of $h$.

Proof. Let $h \in E_{+}$and let $\phi$ be the unique extremal kernel in $L_{1}$ representing $h$. Then

$$
\begin{array}{rlr}
h(0) & =\frac{1}{2 \pi i} \int_{|w|=1} \phi(w) d w=\frac{1}{2 \pi} \int_{0}^{2 \pi} \phi(w) d w \theta \leqq \frac{1}{2 \pi} \int_{0}^{2 \pi}|\phi(w)| d \theta \\
& =\|\phi\|=\|h\|=h(0), & \left(w=e^{i \theta}\right)
\end{array}
$$

and so $w \phi(w) \geqq 0$ almost everywhere.

Conversely, if $w \phi(w) \geqq 0$ almost everywhere then

$$
\|h\| \geqq|h(0)|=\frac{1}{2 \pi} \int \phi(w) w d \theta=\frac{1}{2 \pi} \int|\phi(w)| d \theta \geqq\|h\|,
$$

the last inequality following from the definition of norm (33), and so $h \in E_{+}$ which completes the proof.

Suppose $h$ can be represented by an exponential sum with non-negative coefficients

$$
h(z)=\sum a_{n} \exp \left(\alpha_{n} z\right), \quad a_{n} \geqq 0(n=1,2, \cdots) .
$$

Then one sees easily that $h \in E_{+}$. We do not know whether the converse is true. However, the representation (39) is never unique when it does exist.

Indeed, Wolff [21] proved that if a set of disjoint circles is removed from the unit circle so that only a set of measure zero remains, then

$$
1=\sum r_{n}^{2} \exp \left(\alpha_{n} z\right)
$$

where $r_{n}$ denotes the radius of the $n$th removed circle and $\alpha_{n}$ is its center. By applying a bilinear transformation to this example (see the corollary to Lemma 3 ) we may represent any given exponential $e^{\alpha z}(|\alpha|<1)$ using the same coeffcients and different exponents. It follows from this that if a function has a representation (39) then it has uncountably many such representations.

REMARK 11. If $h \in E$ has a minimizing sequence of exponents and coeffcients, i.e. if there exist $\left\{a_{n}\right\},\left\{\alpha_{n}\right\}$ such that $h(z)=\sum a_{n} \exp \left(\alpha_{n} z\right)$ and $\sum\left|a_{n}\right|=\|h\|$, then signum $a_{n}=$ const., and $|h(0)|=\|h\|$. 
Indeed, let $f \in H_{\infty},\|f\|=1$, be such that $(h, f)=\|h\|$. Then: $\|h\|=(h, f)$ $=\sum a_{n} f\left(\alpha_{n}\right) \leqq \sum\left|a_{n} f\left(\alpha_{n}\right)\right| \leqq \sum\left|a_{n}\right|=\|h\|$. Hence $\left|f\left(\alpha_{n}\right)\right|=1$, and so by the maximum modulus theorem $f=$ const. Therefore, signum $a_{n}=$ const., and $|h(0)|=\sum\left|a_{n}\right|$.

We now consider a different representation of the space $E(G)$. Let $G$ be an arbitrary Jordan domain. Let $M(G)$ be the set of all those entire functions $h(z)$ that can be represented in the form

$$
h(z)=\int e^{z w} d \mu(w)
$$

where $\mu$ is a Borel measure in $G$. We define a norm in $M(G)$ by

$$
\|h\|=\inf \operatorname{Var}(\mu)
$$

taken over all representations (40) of $h$.

THEOREM 9. The two spaces $E(G)$ and $M(G)$ with the norms (15) and (41) are identical.

Proof. Clearly $E(G) \subset M(G)$ and $\|h\|_{M} \leqq\|h\|_{E}$ for all $h \in E(G)$.

To prove the converse, let $h \in M(G)$. We first consider the case where $h$ can be represented in (40) by a measure $\mu$ whose support is entirely inside $G$. Since the support (the smallest closed set containing all the mass) is a closed set, it is at a positive distance from the boundary of $G$. Hence the Borel transform of $h$ is analytic on the boundary of $G$, and therefore by a result of Wolff [21], $h \in E(G)$.

To estimate the norm of $h$, let $\left\{\mu_{n}\right\}$ be a sequence of measures in $G$, each consisting of a finite number of mass points, such that: $\operatorname{Var}\left(\mu_{n}\right)=\operatorname{Var}(\mu)$, and $\int f d \mu_{n} \rightarrow \int f d \mu$ for all $f \in H_{\infty}(G)$. Let $h_{n}(z)=\int e^{z w} d \mu_{n}(w)$. Then $h_{n}$ is a finite exponential sum and therefore $h_{n} \in E(G)$. Also, $\left(h_{n}, f\right) \rightarrow(h, f)$ for each $f \in H_{\infty}(G)$. Hence, $\|h\|_{E} \leqq \lim \inf \left\|h_{n}\right\|_{E} \leqq \operatorname{Var}(\mu)$. (If $E$ were known to be weakly sequentially complete then Wolff's result would not be needed.)

Now let $h \in M(G)$ be arbitrary. Let $\sigma$ be any measure in $G$ that represents $h$. Since $G$ is an open set, there are compact sets $C_{1} \subset C_{2} \subset \cdots$ whose union is $G$. Let $\sigma_{1}$ be the restriction of $\sigma$ to $C_{1}$, and for $n>1$ let $\sigma_{n}$ be the restriction of $\sigma$ to $C_{n} \backslash C_{n-1}$. Then $\sum \operatorname{Var}\left(\sigma_{n}\right)=\operatorname{Var}(\sigma)$.

Let $h_{n}(z)=\int e^{z w} d \sigma_{n}(w)$. Then $\left\|h_{n}\right\|_{M} \leqq \operatorname{Var}\left(\sigma_{n}\right)$ and therefore $h=\sum h_{n}$, the series converging in $M(G)$, and also converging uniformly on compact subsets of the plane. By our previous result, $h_{n} \in E(G)$ and $\left\|h_{n}\right\|_{E} \leqq \operatorname{Var}\left(\sigma_{n}\right)$. Hence the series converges in $E(G)$. Therefore $h \in E(G)$ and $\|h\|_{E} \leqq \operatorname{Var}(\sigma)$. Consequently $\|h\|_{E} \leqq \inf \operatorname{Var}(\sigma)=\|h\|_{M}$. This completes the proof.

5. Additional properties of the space $E$. In this section we work entirely in the unit circle and accordingly we write $E$ instead of $E(G)$. We do not know whether $E$ has a Schauder basis or not, but we shall show that the Taylor series is not such a basis. 
THEOREM 10. There is an $h \in E$ whose Taylor series does not converge in the norm of $E$.

Proof. Let $T_{n}$ be the projection operator sending each $h \in E$ on to the $n$th partial sum of its Taylor series. If the Taylor series always converged the $T_{n}$ would be uniformly bounded. Since $\left\|e^{\alpha z}\right\|=1$ for $|\alpha|<1$ we have:

$$
\left\|T_{n}\right\| \geqq\left\|T_{n} e^{\alpha z}\right\|=\left\|\sum_{0}^{n} \alpha^{k} z^{k} / k !\right\|=\sup \left|\sum_{0}^{n} B_{k} \alpha^{k}\right|
$$

where the supremum is over all $f(z)=\sum B_{k} z^{k} \in H_{\infty}$ such that $\|f\| \leqq 1$. The last equality is merely the statement that $\|h\|=\sup |(h, f)|$.

Since $\alpha$ is arbitrary inside the unit circle we have

$$
\left\|T_{n}\right\| \geqq \sup \left|\sum_{0}^{n} B_{k}\right|,
$$

and by a result of Landau $[10$, pp. 26-27] this is asymptotic to $(1 / \pi) \log n$, which completes the proof.

COROLlaRY. There are functions $h(z)=\sum A_{n} z^{n} / n ! \in E$ and $f(z)=\sum B_{n} z^{n}$ $\in H_{\infty}$ for which the series $\sum A_{n} B_{n}$ is not convergent.

Proof. It is known (Day [4, p. 69]) that a weak Schauder basis is a strong basis. Hence the Taylor series is not weakly convergent for all $h \in E$. QED.

The series is of course always Abel summable (see (30)).

Problem (TAYloR $\left[18\right.$, p. 33]). If $\left\{A_{n}\right\}$ has the property that $\lim \sum A_{n} B_{n} r^{n}\left(r \rightarrow 1^{-}\right)$exists for all $f(z)=\sum B_{n} z^{n} \in H_{\infty}$, does it follow that $h(z)=\sum A_{n} z^{n} / n$ ! is in $E$ ? This would follow if it were known that $E$ is weakly sequentially complete.

The space $H_{\infty}$ may be considered as a space of linear operators on $E$. Indeed, if $f \in H_{\infty}$ and $h(z)=\sum a_{n} \exp \left(\alpha_{n} z\right) \in E$ then we define the operator $T_{f}: E \rightarrow E$ by:

$$
\left(T_{f} h\right)(z)=\sum a_{n} f\left(\alpha_{n}\right) \exp \left(\alpha_{n} z\right) .
$$

$T_{f}$ may also be considered as an infinite order differential operator: if $f(z)$ $=\sum B_{n} z^{n}$ and $D=d / d z$ then formally $T_{f}=\sum B_{n} D^{n}$.

In analogy to the problem stated above we may ask: if $h$ belongs to the domain of all the operators $T_{f}\left(f \in H_{\infty}\right)$, must $h \in E$ ?

The adjoint operator $T_{f}^{*}: H_{\infty} \rightarrow H_{\infty}$ is simply multiplication by $f$.

$$
\left(T_{f}^{*} g\right)(z)=f(z) g(z) \text {. }
$$

REMARK 12. If $f\left(e^{i \theta}\right)$ is essentially bounded away from zero (i.e. if $\left|f\left(e^{i \theta}\right)\right|$ $\geqq c>0$ almost everywhere), then $T_{j}$ is onto.

Indeed, if $h$ is given by (32) let $h_{1}(z)$ be represented by $\phi(w) / f(w)$. Then $T_{f} h_{1}=h$. 
REMARK 13. If $f$ is analytic for $|z| \leqq 1$ and has no zeros for $|z|<1$, then $T_{f}$ is one-to-one.

Indeed, let $h \in E$ and assume $T_{f} h=0$. For any $\epsilon>0, h$ maybe expressed in terms of its Borel transform (4) with $r=1+\epsilon$. Then

$$
\left(T_{f} h\right)(z)=\frac{1}{2 \pi i} \int_{|w|=1+\epsilon} e^{z w} f(w) H(w) d w \equiv 0,
$$

where $\epsilon$ is chosen so that $f$ is analytic on $|w|=1+\epsilon$. From (44) it then follows that $f(w) H(w)(|w|=1+\epsilon)$ are the boundary values of a function analytic for $|w|<1+\epsilon$. Since $H$ is analytic for $|w|>1$, and $f$ has no zeros for $|w|<1$, it follows that $H$ can be at worst a rational function with poles only on $|w|=1$. But then $h$ would have the form $\sum P_{k}(z) \exp \left(w_{k} z\right)$, where the $P_{k}$ are polynomials, and $\left|w_{k}\right|=1$, and such a function is not in $E$ (for example, if $H(w)=1 /(w-1)$ then $h(z)=e^{z}$. Thus $H$ has no poles at all, and therefore $H=0$.

\section{BIBLIOGRAPHY}

1. S. Banach, Théorie des opérations linéaires, Warszawa-Lwow, 1932.

2. L. Bieberbach, Lehrbuch der Funktionentheorie, vol. II, Leipzig-Berlin, 1927.

3. R. P. Boas, Entire functions, New York, Academic Press, 1954.

4. M. M. Day, Normed linear spaces, Ergebnisse der Mathematik, vol. 21, Berlin, SpringerVerlag, 1958.

5. A. Denjoy, Sur les singularités des séries de fractions rationnelles, Rend. Circ. Mat. Palermo vol. 50 (1926) pp. 1-95.

6. - Sur les séries de fractions rationnelles, Bull. Soc. Math. France vol. 52 (1924) pp. $418-434$.

7. Tord Hall, On polynomials bounded at an infinity of points, Thesis, Uppsala, Appelbergs Boktryckeri, 1950.

8. S. Ya. Havinson, On some extremal problems of the theory of analytic functions, Moscov. Gos. Univ. Uč. Zap. vol. 148 (1951), Matematika vol. 4, pp. 133-143 (Russian).

9. E. Hille and R. S. Phillips, Functional analysis and semi-groups, Amer. Math. Soc. Colloquium Publications, vol. 31, 1957.

10. E. Landau, Darstellung und Begründung einiger neuerer Ergebnisse der Funktionentheorie, 2d ed., 1929.

11. B. Ya. Levin, Distribution of the roots of entire functions, Moscow, 1956 (Russian).

12. A. J. Macintyre and W. W. Rogosinski, Extremum problems in the theory of analytic functions, Acta Math. vol. 82 (1950) pp. 275-325.

13. S. N. Mergelyan, Uniform approximations to functions of a complex variable, Uspehi Mat. Nauk vol. 7, no. 2 (1952) pp. 31-122; Amer. Math. Soc. Translation, no. 101.

14. R. Nevanlinna, Eindeutige analytische Funktionen, Berlin, 1936.

15. I. I. Privaloff, Boundary properties of analytic functions, Moscow-Leningrad, 1950 (Russian); Deutscher Verlag der Wissenschaften, Berlin, 1956 (German translation).

16. W. W. Rogosinski and H. S. Shapiro, On certain extremum problems for analytic functions, Acta Math. vol. 90 (1953) pp. 287-318.

17. J. E. Scroggs, Invariant subspaces of a normal operator, Duke Math. J. vol. 26 (1959) pp. $95-112$.

18. A. E. Taylor, Banach spaces of functions analytic in the unit circle II, Studia Math. vol. 12 (1951) pp. 25-50. 
19. J. L. Walsh, Über die Entwicklung einer analytischen Funktion nach Polynomen, Math. Ann. vol. 96 (1926) pp. 430-436.

20. J. Wermer, On invariant subspaces of normal operators, Proc. Amer. Math. Soc. vol. 3 (1952) pp. 270-277.

21. J. Wolff, Sur les séries $\sum A_{k} /\left(z-\alpha_{k}\right)$, C. R. Acad. Sci. Paris vol. 173 (1921) pp. 1057$1058,1327-1328$.

Wayne State University,

Detroit, Michigan

UNIVERSITY OF MiCHIGAN,

ANn Arbor, Michigan

TÜBINGEN UNIVERSITY,

TÜBINGEN, GERMANY 